XI.

Aus dem Pharmakologisehen Institut der Universität Berlin.

\title{
Über den Einfluß von Atropin und Pilokarpin auf die Antikörperbildung bei Kaninchen.
}

Von

Georg Joachimoglu und Yoshitsune Wada.

(Mit 2 Kurven.)

Über die Natur der Antikörper sind wir mangelhaft unterrichtet. Wir wissen nar, daß sie nach parenteraler Einverleibung gewisser Stoffe entstehen, über deren Zusammensetzung wir ebenfalls nicht viel wissen. Diese Stoffe nennen wir Antigene, weil sie zu einer Antikörperbildang führen. Das Wesen beider Gruppen von Körpern ist uns unbekannt. Nur ihre Wirkungen kennen wir. Man nimmt weiter an, daß die Antikörper in den sogenannten hämatopoetischen Organen entstehen und faßt den Vorgang als eine Abwehrwirkung des Organismus, als eine Entgiftung eines Giftes auf. Dabei hat man zu unterscheiden Bildung der Antikörper und Transport derselben in das Blat, wo sie am leichtesten nachznweisen sind. Dieser Transport oder Abgabe an das Blnt erinnert in mancher Hinsicht an die Sekretion von Stoffen aus Driisen. Die Angabe von Salomonsen und Madsen ${ }^{1}$, die gefunden haben, daß bei einem Pferd, welches längere Zeit mit Diphtherietoxin behandelt worden war, der Antitoxingehalt des Serums nach intravenöser Injektion von 1,4 g Pilokarpin deutlich zunimmt, ist in dieser Hinsicht von prinzipieller Bedeutung. Ist diese Wirkung des Pilokarpins mit seinen tubrigen pharmakologischen Wirkungen auf das parasympathische Nervensystem in Parallele zu setzen, so muß erwartet werden, daß Atropin die entgegengesetzte Wirkung zeigt. Die genannten Autoren konnten

1) Compt. rend. de l'Académie des Sciences 1898, Bd. 126, S. 1229. 
nun durch Injektion von insgesamt $0,7 \mathrm{~g}$ Atropin eine Abnahme des Antitoxingehaltes des Serums bei demselben Pferd nicht feststellen.

Der antitoxische Gehalt des Serums entsprach vor der Atropininjektion 30 Einheiten pro Kubikzentimeter. 5 Stunden nach der Atropinzufuhr sowie am nächsten und übernächsten Tag wurde derselbe Wert ermittelt. Die intravenöse Injektion von $1,4 \mathrm{~g}$ Pilokarpin steigerte dagegen den Antitoxingehalt von 25 auf 30 im ersten Versuch, während in einem zweiten Versuch kein Einfluß festgestellt werden konnte.

Ein dritter Versuch verlief folgendermaßen:

\begin{tabular}{|c|c|c|c|}
\hline Datum & Zeit & $\begin{array}{l}\text { Immunitäts- } \\
\text { einheiten } \\
\text { pro ccm }\end{array}$ & Bemerkungen \\
\hline 11. X. & - & $4 \check{\jmath}$ & - \\
\hline 18. X. & 一 & 40 & - \\
\hline 25. X. & - & 35 & - \\
\hline 28. X. & - & 30 & Unmittelbar vor der ersten Injektion. \\
\hline & $12^{\mathrm{h}} 40^{\prime}$ & - & Injektion von $20 \mathrm{mg}$ Pilokarpin. \\
\hline & $12^{\mathrm{h}} 42^{\prime}$ & 35 & - \\
\hline & $12^{\mathrm{h}} 47^{\prime}$ & 35 & - \\
\hline & $12^{\mathrm{h}} 55^{\prime}$ & - & Injektion von $80 \mathrm{mg}$ Pilokarpin (insgesamt). \\
\hline & $1^{\text {h }} 05^{\prime}$ & 40 & Speichelfluß sehr stark. \\
\hline & $2^{\mathrm{h}} 05^{\prime}$ & 30 & Speichelfluß vermindert. \\
\hline & $5^{\mathrm{h}} 30^{\prime}$ & 30 & Speichelfuß ganz aufgehört. \\
\hline 29. X. & - & 25 & - \\
\hline 30.X. & - & 25 & - \\
\hline
\end{tabular}

Wir sehen aus diesem Versuch, daß der antitoxische Gehalt des Serums großen Schwankangen unterworfen war. Zwischen dem 11. und 28. X. schwankte er um 15 Einheiten. Hätten die genannten Forscher etwa am 28. X. Atropin injiziert, so wären sie zu dem Resultat gekommen, daB dieses Gift eine Abnahme des Antitoxingehaltes hervorruft. Diese Überlegung zeigt uns, wie vorsichtig man bei der Beurteilung der Befunde sein muß.

Aus diesen Untersuchungen ist jedenfalls geschlossen worden, daB das Pilokarpin eine Zunahme des Antitoxingehaltes bedingt. Wir hätten es demnach hier mit einer neuen pharmakologischen Wirknng des Pilokarpins zu tan, die durch Atropin nicht beeinflußt wird.

Dieser Gesichtspankt schien uns vom pharmakologischen Standpunkt aus immerhin interessant genug, um eine Nachprufung dieser Befunde vorzunehmen. 
Die Versuche wurden an Kaninchen ansgefübrt, die mit abgetöteten Typhusbakterien (1 Stunde bei $60^{\circ}$ ) intravenös immunisiert wurden.

\section{Atropinversuche.}

Versuch 1.

Drei Kaninchen erhalten, nachdem die normale Agglutination (Tsbelle 1) festgestellt wurde, intravenös $1 / 2$ Öse Typhusbakterien.

Tabelle 1.

\begin{tabular}{c|c|c|c|c}
\hline \multirow{2}{*}{ Datum } & \multirow{2}{*}{ Verdünnung } & \multicolumn{3}{|c}{ Körpergewicht } \\
& & Nr. 33 & Nr. 34 & Nr. 35 \\
& & $2,2 \mathrm{~kg}$ & $2,2 \mathrm{~kg}$ & $2,2 \mathrm{~kg}$ \\
\hline \multirow{2}{*}{ 30. XI. } & $1: 5$ & 0 & + & 0 \\
& $1: 10$ & 0 & + & 0 \\
& $1: 20$ & 0 & 0 & 0
\end{tabular}

Nach 7 Tagen ergab die Pruffung der Agglutination folgendes:

Tabelle 2 .

\begin{tabular}{c|c|c|c|c}
\hline Datum & Verdünung & Nr. 33 & Nr. 34 & Nr. 35 \\
\hline 7. XII. & $1: 80$ & + & + & + \\
& $1: 160$ & + & + & + \\
& $1: 200$ & + & + & + \\
$1: 260$ & + & + & + \\
& $1: 320$ & + & + & + \\
& $1: 400$ & + & + & + \\
$1: 530$ & + & + & 0 \\
& $1: 640$ & + & + & 0 \\
& $1: 800$ & + & 0 & 0 \\
& $1: 1060$ & + & 0 & 0 \\
$1: 1280$ & + & 0 & 0 \\
& $1: 1600$ & + & 0 & 0 \\
& $1: 2130$ & 0 & 0 & 0 \\
& $1: 2560$ & 0 & 0 & 0
\end{tabular}

Die drei Kaninchen erhielten nun intravenös $23 \mathrm{mg}$ Atropinsulfat pro Kilo ( $5 \%$ ige Lösung).

Die intravenöse Injektion des Atropins schien uns am zweckmäßigsten $\mathrm{zu}$ sein, weil die Untersuchnngen von A. Heffter und G. Fickewirth ${ }^{1}$ ) gezeigt haben, daß die bekannte Resistenz des Kaninchens gegen Atropin für die stomachale und subkutane Applika-

1) Biochem. Zeitschr. 1912, Bd. 40, S. 36 . 
tion, aber nicht für die intravenöse Darreichung besteht. Die Prífung der Agglutination 2 Stunden nach der Injektion des Atropins ergab folgendes:

Tabelle 3.

\begin{tabular}{c|c|c|c|c}
\hline Datum & Verdünnung & Nr. 33 & Nr. 34 & Nr. 35 \\
\hline 7. XII. & $1: 160$ & + & + & + \\
& $1: 200$ & + & + & + \\
& $1: 260$ & + & + & + \\
& $1: 320$ & + & + & + \\
$1: 400$ & + & + & 0 \\
& $1: 530$ & + & + & 0 \\
$1: 640$ & + & + & 0 \\
& $1: 800$ & + & 0 & 0 \\
& $1: 1060$ & + & 0 & 0 \\
$1: 1280$ & + & 0 & 0 \\
$1: 1600$ & 0 & 0 & 0 \\
& $1: 2130$ & 0 & 0 & 0 \\
$1: 2560$ & 0 & 0 & 0 \\
& $1: 5120$ & 0 & 0 & 0
\end{tabular}

Dieser Versuch zeigt uns, daß bei dem einen Kaninchen (Nr. 33) eine geringe Abnahme des Agglutiningehaltes des Serums eingetreten ist, ebenso verhielt sich das Tier Nr. 35, das an sich wenig Agglutinine gebildet hatte, während bei dem Tier Nr. 34 der Titer unverändert geblieben ist. Man kann auf Grund dieses Befundes von einer Wirkung des Atropins kaum sprechen.

Versuch 2.

Die normale Agglatination ergab auch bei der Verdünnung $1: 5$ ein negatives Resultat. 10. XII. Injektion von Typhusbazillen wie in Versuch 1. Die Prlifung der Agglutination am 16. XII. vor und 2 stunden nach intravenöser Injektion von $33 \mathrm{mg}$ Atropin pro Kilo ergab folgendes:

Tabelle 4.

\begin{tabular}{c|c|c|c}
\hline \multirow{2}{*}{ Datum } & Verdünnung & \multicolumn{2}{|c}{$\begin{array}{r}\text { Körpergewicht Nr. 38 2,4 kg } \\
\text { vor der Injektion }\end{array}$} \\
& & nach der Injektion \\
\hline \multirow{2}{*}{ 16. XII. } & $1: 160$ & + & + \\
& $1: 320$ & + & + \\
& $1: 400$ & + & + \\
& $1: 530$ & + & + \\
& $1: 640$ & + & + \\
& $1: 800$ & + & + \\
$1: 1060$ & 0 & 0 \\
& $1: 1280$ & 0 & 0
\end{tabular}


Das Tier weist hier nach einer großen Atropindosis eine geringe Zunahme des Agglatiningehaltes auf. Zwei andere Tiere, die intravenös mit dieser großen Atropindosis behandelt wurden, starben akat. Von einer Prüfung des Agglutiningehaltes wurde deswegen abgesehen.

Eine andere Versuchsreihe mit einer Dosis von $33 \mathrm{mg}$ Atropin pro Kilo ergab keinen Unterschied des Agglntiningehaltes vor und nach der Injektion. Hier ist das Blut 1 Stunde vor und nach der Injektion entnommen worden. Zur Kontrolle haben wir gleichzeitig einem anderen Tier Kochsalzlösung injiziert, wodureh der Agglutiningehalt ebenfalls gar keine Veränderung erfuhr.

\section{Versuch 3.}

In diesem Versuch erhielten die Tiere nach der Injektion von Typhusbakterien täglich kleine Atropinmengen subkutan bzw. intravenōs. Kaninchen Nr. 1-6 erhalten am 7. IX. Typhusbakterien. Kaninchen Nr. 1 und 2 täglich $20 \mathrm{mg}$ Atropin intravenös, Nr. 3 und 4 subkutan $50 \mathrm{mg}$ Atropin. Nr. 5 und 6 dienten als Kontrollen. Die Prüfung der Agglntination am 14. IX. ergab folgendes:

Tabelle 5.

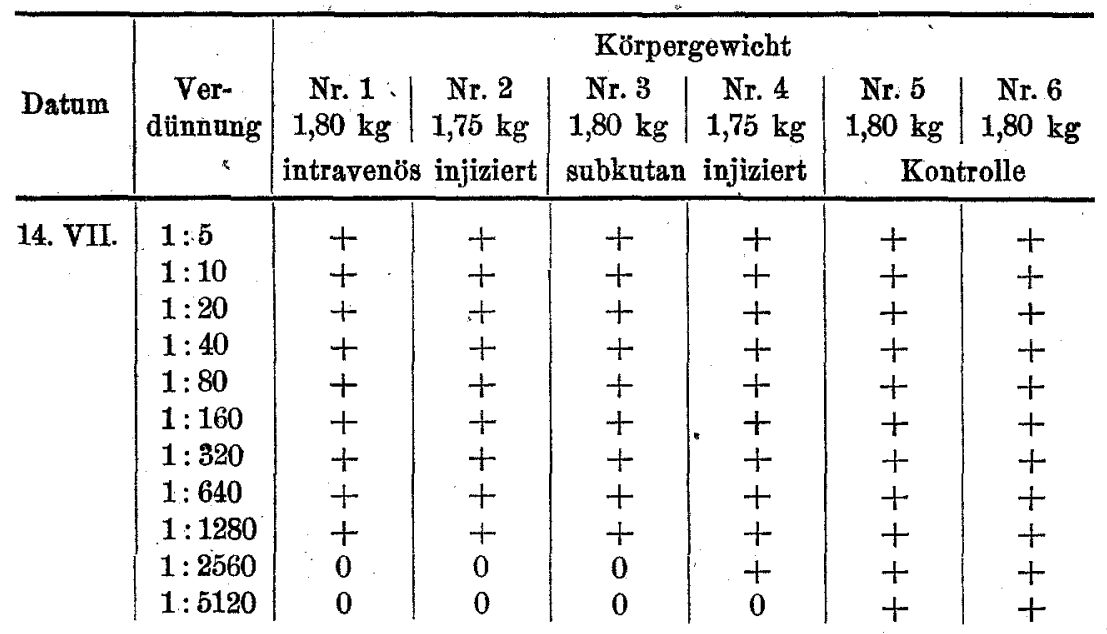

Die Tabèlle zeigt uns, daß die Kontrolltiere einen erheblich höheren Agglutiningehalt anfweisen als die mit Atropin behandeltén.

In den nächsten 8 Tagen erhielten die Tiere, nachdem sie am 14. IX. eine 2. Injektion von Typhusbazillen erbalten hatten, täglich die gleiche Menge Atropin. Die Agglutinationsprufung am 21. IX. ergab folgendes: 
Tabelle 6.

\begin{tabular}{l|c|c|c|c|c|c}
\hline Datum & Verdünnung & $\begin{array}{c}\text { Nr. 1 } \\
\text { intravenös injiziert }\end{array}$ & $\begin{array}{c}\text { Nr. 2 } \\
\text { subkatan }\end{array}$ & $\begin{array}{c}\text { Nr. 3 } \\
\text { injiziert }\end{array}$ & $\begin{array}{c}\text { Nr. 5 } \\
\text { Kontrolle }\end{array}$ \\
\hline \multirow{2}{*}{ 21. IX. } & $1: 40$ & + & + & + & + & + \\
& $1: 80$ & + & + & + & + & + \\
& $1: 160$ & + & + & + & + & + \\
$1: 320$ & + & + & + & + & + \\
& $1: 640$ & + & + & + & + & + \\
$1: 1280$ & + & + & + & + & + \\
& $1: 2560$ & \pm & + & + & + & + \\
$1: 5120$ & 0 & + & \pm & + & + \\
& $1: 10240$ & 0 & + & 0 & 0 & 0 \\
& $1: 20480$ & 0 & + & 0 & 0 & 0 \\
& $1: 40960$ & 0 & \pm & 0 & 0 & 0
\end{tabular}

Tier Nr. 6 starb am 17. IX.

Die Tabelle zeigt hier zwischen Kontroll- und Atropintieren keinen wesentlichen Unterschied, nur das Tier Nr. 2, welches intravenös Atropin erhalten hatte, wies einen sehr hohen Agglutinationstiter $(1: 20000)$ auf. Es handelt sich wohl hier um eine zufällige Erscheinung, denn sonst ist niemals bei unseren Tieren ein so hoher Agglutiningehalt beobachtet worden. Am 21. IX. erhielten die Tiere eine 3. Injektion von Typhusbakterien und in der darauffolgenden Woche täglich Nr. 1 und 2 intravenös je $40 \mathrm{mg}$ Atropin, Nr. 3 und 4 subkutan je $80 \mathrm{mg}$. Die Agglutinationsprüfung am 28. IX. ergab folgendes:

Tabelle 7.

\begin{tabular}{l|c|c|c|c|c}
\hline Datum & Verdïnung & \multicolumn{2}{|c|}{$\begin{array}{c}\text { Nr. 1 } \\
\text { intravenös injiziert }\end{array}$} & $\begin{array}{c}\text { Nr. 2 } \\
\text { subkutan injiziert }\end{array}$ & $\begin{array}{c}\text { Nr. 4 } \\
\text { Kontrolle }\end{array}$ \\
\hline \multirow{2}{*}{ 28. IX. } & $1: 80$ & + & + & + & + \\
& $1: 160$ & + & + & + & + \\
& $1: 320$ & + & + & + & + \\
$1: 640$ & + & + & + & + \\
$1: 1280$ & + & + & + & + \\
& $1: 2560$ & 0 & + & + & + \\
& $1: 5120$ & 0 & + & + & + \\
& $1: 10240$ & 0 & 0 & + & + \\
& $1: 20480$ & 0 & 0 & 0 & 0
\end{tabular}

Tier Nr. 3 starb in der Zwischenzeit.

Den höchsten Agglutiningehalt weist hier das Kontrolltier Nr. 5 und das subkutan injizierte (Nr. 4) auf, während die intravenös inji- 
zierten Tiere (Nr. 1 und 2) einen erheblich schwächeren Agglutiningehalt anfweisen. Man wäre geneigt, aus diesen Versuchen die Tat\$ache zu entnehmen, dal die chronische Darreichung von Atropin den Agglutiningehalt des Serums herabsetzt. Die Wiederholung dieses Versuchs fuhrte jedoch zu einem anderen Riesultat.

\section{Versuch 4.}

Sechs Kaninchen erbjelten am 10. X., nachdem die normale Agglntination geprüft war (Tabelle 8), intravenös Typhusbakterien wie in Versuch 1.

Tabolle 8.

\begin{tabular}{c|c|c|c|c|c|c|c}
\hline \multirow{2}{*}{ Datum } & $\begin{array}{c}\text { Ver- } \\
\text { dünnung }\end{array}$ & $\begin{array}{c}\text { Nr. 15 } \\
2,25 \mathrm{~kg}\end{array}$ & $\begin{array}{c}\text { Nr. 16 } \\
2,10 \mathrm{~kg}\end{array}$ & $\begin{array}{c}\text { Nr. 17 } \\
2,15 \mathrm{~kg}\end{array}$ & $\begin{array}{c}\text { Nr. 18 } \\
2,25 \mathrm{~kg}\end{array}$ & $\begin{array}{c}\text { Nr. 19 } \\
2,05 \mathrm{~kg}\end{array}$ & $\begin{array}{c}\text { Nr. 20 } \\
2,25 \mathrm{~kg}\end{array}$ \\
\hline \multirow{2}{*}{$10 . \mathrm{X}}$. & $1: 5$ & + & + & 0 & 0 & + & + \\
& $1: 10$ & + & + & 0 & 0 & + & + \\
& $1: 20$ & 0 & + & 0 & 0 & 0 & 0
\end{tabular}

Drei dieser Kaninchen Nr. 18, 19 und 20 erhielten nan täglicb $20 \mathrm{mg}$ Atropin intravenös. Am 18. X. ergab die Agglutinationsprüfung:

Tabelle 9.

\begin{tabular}{l|l|c|c|c|c|c|c}
\hline \multirow{2}{*}{ Datum } & Ver- & \multicolumn{3}{|c|}{ Kontrolliere } & \multicolumn{4}{c}{ Atropintiere } \\
& diinnung & Nr. 15 & Nr. 16 & Nr. 17 & Nr. 18 & Nr. 19 & Nr. 20 \\
\hline \multirow{2}{*}{ 18. X. } & $1: 40$ & + & + & + & + & + & + \\
& $1: 80$ & + & + & + & + & + & + \\
& $1: 160$ & + & + & + & + & + & + \\
& $1: 320$ & + & + & + & + & + & + \\
$1: 640$ & + & + & + & + & + & + \\
& $1: 1280$ & + & + & + & + & + & + \\
$1: 2560$ & + & + & + & + & + & + \\
& $1: 5120$ & 0 & + & 0 & 0 & 0 & 0 \\
$1: 10240$ & 0 & 0 & 0 & 0 & 0 & 0
\end{tabular}

Wir sehen hier, daß zwischen Atropintieren und Kontrolltieren kaum ein Unterschied des Agglutiningehaltes festzustellen ist. Am 18. $X$. erbielten die Tiere eine zweite Antigeninjektion and in der darauffolgenden Woche Tier Nr. 18-20 täglich $40 \mathrm{mg}$ Atropin intravenös. Die Agglutinationsprüfung am 25. X. ergab: 
Tabelle 10.

\begin{tabular}{l|c|c|c|c|c|c|c}
\hline \multirow{2}{*}{ Datum } & Ver- & \multicolumn{3}{|c|}{ Kontrolltiere } & \multicolumn{3}{|c}{ Atropintiere } \\
& dïnnung & Nr. 15 & Nr. 16 & Nr. 17 & Nr. 18 & Nr. 19 & Nr. 20 \\
\hline 25. X. & $1: 160$ & + & + & + & + & + & + \\
& $1: 320$ & + & + & + & + & + & + \\
& $1: 640$ & + & + & + & + & + & + \\
& $1: 1280$ & + & + & + & + & + & + \\
& $1: 2560$ & + & + & + & + & + & + \\
& $1: 5120$ & + & + & + & + & + & + \\
& $1: 10240$ & + & + & 0 & + & + & 0 \\
& $1: 20480$ & 0 & 0 & 0 & 0 & 0 & 0
\end{tabular}

Auch hier konnte ein Untersehied zwischen Atropin- und Kontrolltieren nicht festgestellt werden. Tier Nr. 17 und 19 starben in den nächsten Tagen und blieben deshalb unberücksichtigt. Am 25. X. bekamen die übrigen Tiere eine 3. Antigeninjektion und in der darauffolgenden Woche täglich $60 \mathrm{mg}$ Atropin intravenös. Die Agglutination am 1. XI. ergab folgendes:

Tabelle 11.

\begin{tabular}{l|l|c|c|c|c}
\hline \multirow{2}{*}{ Datum } & \multirow{2}{*}{ Verdünnung } & \multicolumn{2}{|c|}{ Kontrolltiere } & \multicolumn{2}{|c}{ Atropintiere } \\
& & Nr. 15 & Nr. 16 & Nr. 18 & Nr. 20 \\
\hline \multirow{2}{*}{ 1. XI. } & $1: 160$ & + & + & + & + \\
& $1: 3: 0$ & + & + & + & + \\
& $1: 640$ & + & + & + & + \\
& $1: 1280$ & + & + & + & + \\
& $1: 2560$ & + & + & + & + \\
& $1: 5120$ & + & + & + & 0 \\
& $1: 10240$ & + & + & + & 0 \\
& $1: 20480$ & + & 0 & + & 0 \\
& $1: 40960$ & 0 & 0 & 0 & 0
\end{tabular}

Zwischen dem Kontrolltier Nr. 15 und dem Atropintier Nr. 18 ist kein Unterschied im Agglutiningehalt. Auch bei den anderen Tieren ergeben sich geringfügige Unterschiede. Nimmt man die Mittelzahlen aus den vorhergehenden Tabellen dieser Versuchsreihe und trägt sie in ein Koordinatensystem ein, and zwar in die $A b-$ szisse die Versuchstage, in die Ordinate die Verdünnung des Serums, die eine positive Agglutination zeigt, so kommen wir zu einer Kurve, 
die uns zeigt, daß die Atropintiere in der 3. Versuchswoche weniger Agglutinine anfweisen als die Kontrolltiere.

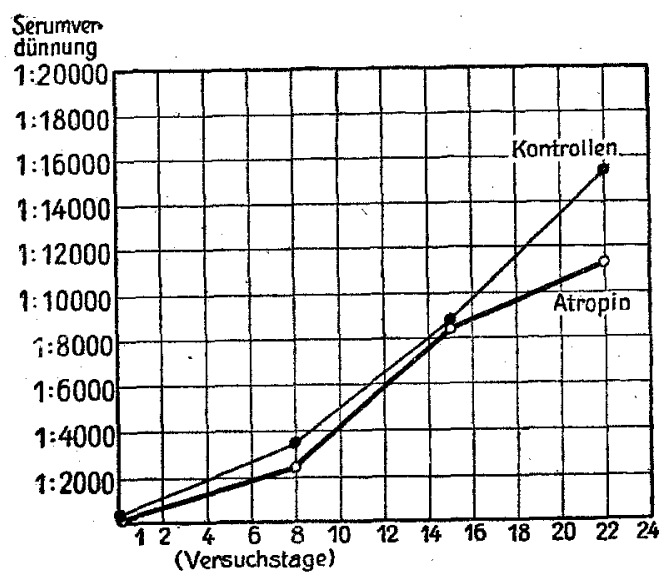

Kurve 1.

Richtiger scheint uns jedoch, die relative Steigerung des Agglutiningehalts gegen die Werte des Normalserums zu vergleichen. Das geht aus folgender Tabelle hervor:

Tabelle 12.

\begin{tabular}{c|r|r|r|r|r|r}
\hline \multirow{2}{*}{$\begin{array}{c}\text { Relative Steige- } \\
\text { rung am Ende }\end{array}$} & \multicolumn{3}{|c|}{ Kontrolltiere } & \multicolumn{3}{|c}{ Atropintiere } \\
\hline Nr. 15 & Nr. 16 & Nr. 17 & Nr. 18 & Nr. 19 & Nr. 20 \\
\hline der 1. Woche & 256 & 256 & 2560 & 2560 & 256 & 256 \\
2. & 1024 & 512 & 5120 & 10240 & 1024 & 512 \\
3. & 2048 & 516 & - & 20480 & - & $\mathbf{2 5 6}$
\end{tabular}

Wir glaubén nicht, daß dieser Befund uns berechtigt, eine Abnahme des Agglutiningehaltes bei den Atropintieren gegenuber den Kontrollen anzunehmen.

\section{Pilokarpinversuche.}

\section{Versuch 1.}

Kaninchen Nr. 38 von 2,4 kg Gewicht erhält am 10. XII. 1921 pro Kilo $1 / 2$ Öse Typhusbazillen. Am 21. XII. Agglutination geprüft und $1,5 \mathrm{ccm}(=15 \mathrm{mg}) 1 \%$ Pilocarpin. hydrochlor. intravenös injiziert. Nach 2 Stunden wieder Titer bestimmt (vgl. Tabelle 13). 
Tabelle 13.

\begin{tabular}{c|c|c|c}
\hline Datum & Verdünnung & Vor der Injektion & Nach der Injektion \\
\hline 21. XII. & $1: 80$ & + & + \\
& $1: 160$ & + & + \\
$1: 320$ & + & + \\
$1: 640$, & + & + \\
$1: 800$ & + & + \\
$1: 1060$ & + & + \\
$1: 1280$ & + & + \\
$1: 1600$ & + & 0 \\
$1: 2130$ & 0 & 0 \\
& $1: 2560$ & 0 & 0
\end{tabular}

Das 2 Stunden nach der Pilokarpinzufuhr entnommene Serum zeigt eine geringe Abnahme des Agglutinintiters.

\section{Versuch 2.}

Zwei Kaninchen erhielten am 1. und 7. XI. Typhusbazillen wie in Versuch 1. Der normale Agglutiningehalt betrug bei beiden Tieren 1:20. Am 11. XI. Agglutination gepriuft. Darnach erhielt das eine Tier zur Kontrolle $1,5 \mathrm{ccm}$ phys. $\mathrm{NaCl}$, das andere Pilokarpin wie in Versuch 1. Nach 2 Stunden 2. Blutentnahme zur Agglutinationsprüfung.

Tabelle 14.

\begin{tabular}{|c|c|c|c|c|c|}
\hline \multirow[b]{2}{*}{ Datum } & \multirow[b]{2}{*}{ Verdünnung } & \multicolumn{2}{|c|}{ Vor der Injektion } & \multicolumn{2}{|c|}{ Nah dar Tnioltion } \\
\hline & & $\begin{array}{c}\mathrm{Nr} .27 \\
1,95 \mathrm{~kg} \mathrm{Gewicht} \\
\mathrm{NaCl}\end{array}$ & $\begin{array}{c}\text { Nr. } 28 \\
1,65 \mathrm{~kg} \text { Gewicht } \\
\text { Pilokarpin }\end{array}$ & $\begin{array}{l}\text { Nach de } \\
\text { Nr. } 27\end{array}$ & $\begin{array}{l}\text { Injektion } \\
\text { Nr. } 28\end{array}$ \\
\hline 11. XI. & $1: 160$ & + & + & + & + \\
\hline & $1: 320$ & + & + & + & + \\
\hline & $1: 640$ & + & + & + & + \\
\hline & $1: 1280$ & + & + & + & + \\
\hline & $1: 2560$ & + & + & + & + \\
\hline & $1: 2800$ & + & + & + & + \\
\hline & $1: 3200$ & + & + & + & + \\
\hline & $1: 3600$ & + & $t$ & + & + \\
\hline & $1: 4200$ & 0 & + & + & + \\
\hline & $1: 5120$ & 0 . & + & + & + \\
\hline & $1: 6400$ & 0 & 0 & 0 & + \\
\hline & $1: 8500$ & 0 & 0 & 0 & $\mathbf{0}$ \\
\hline & $1: 10240$ & 0 & 0 & 0 & $\mathbf{0}$ \\
\hline
\end{tabular}

Beide Tiere zeigen 2 Stunden nach der Kochsalz- bzw. Pilokarpininjektion eine geringe Zunahme dẹ Titers. 


\section{Versuch 3.}

Drei Kaninchen erhielten am 12. XI. 1921 Typhnsbazillen wie in Versuch 1. Am 17. Xr. Agglntinationsprüfung. Zwei davon (Nr. 23 und 26) erhielten darnach je $10 \mathrm{mg}$ Pilocarpin. hydrochl, intravenös. Nach 41/2 Etunden $15 \mathrm{mg}$ Pilocarpin. hydrochl. Das Kontrolltier (Nr. 22) erhieit 1,0 cem $\mathrm{NaCl}$ und nach $4^{1 / 2}$ Stunden $1,5 \mathrm{ccm} \mathrm{NaCl}$. Am nächsten Tag Blutentzabme zur Agglutinationsprifung. Das Ergebnis der Agglutinationsprüfung ist aus folgender Tabelle ersichtlich.

Tabello 15.

\begin{tabular}{|c|c|c|c|c|c|c|c|}
\hline \multirow[b]{2}{*}{ Datum } & \multirow[b]{2}{*}{$\begin{array}{c}\text { Ver- } \\
\text { dünnung }\end{array}$} & \multicolumn{3}{|c|}{ Vor der Injertion } & \multicolumn{3}{|c|}{ Nach der Injektion } \\
\hline & & $\begin{array}{c}\text { Nr. } 22 \\
2,55 \mathrm{~kg} \text { Gew. } \\
\text { Kontrolle }\end{array}$ & $\left\{\begin{array}{c}\text { Nx. } 23 \\
\text { 2,25 kg Gew. } \\
\text { Pilokarpin }\end{array}\right.$ & $\left\{\begin{array}{c}\mathrm{Nr} \cdot 26 \\
2,85 \mathrm{~kg} \text { Gew } \\
\text { Pilokarpin }\end{array}\right.$ & $\begin{array}{l}\text { Nach } \\
\text { Nr. } 22\end{array}$ & $\begin{array}{l}\text { der Ins } \\
\text { Nr. } 23\end{array}$ & Nr. 26 \\
\hline 17. bis & $1: 160$ & $t$ & + & + & + & + & + \\
\hline 18. XI. & $1: 320$ & + & + & + & + & + & + \\
\hline & $1: 640$ & + & + & + & + & + & + \\
\hline & $1: 1280$ & + & $t$ & + & + & + & + \\
\hline & $1: 2560$ & + & + & + & + & + & + \\
\hline & $1: 2840$ & + & $\frac{1}{2}$ & 0 & + & + & + \\
\hline & $1: 3200$ & + & + & 0 & + & + & 0 \\
\hline & $1: 3650$ & + & $t$ & 0 & + & + & 0 \\
\hline & $1: 4200$ & + & + & 0 & + & + & 0 \\
\hline & $1: 5120$ & + & + & 0 & + & + & 0 \\
\hline & $1: 5680$ & + & + & 0 & + & + & 0 \\
\hline & $1: 6400$ & + & + & 0 & + & + & 0 \\
\hline & $1: 7300$ & + & + & 0 & + & + & 0 \\
\hline & $1: 8500$ & + & + & 0 & 0 & + & 0 \\
\hline & $1: 10240$ & 0 & 0 & 0 & 0 & + & 0 \\
\hline & $1: 17000$ & 0 & 0 & 0 & 0 & 0 & 0 \\
\hline
\end{tabular}

Bei dem Kontrolltier ist eine geringe Abnahme, bei den zwei Pilokarpintieren eine geringe Zunahme des Agglutiningehaltes festzustellen.

\section{Versuch 4.}

Zwei Kaninchen erhielten am 8. und 12. XI. 1921 Typhushazillen wie in Versuch 1. Am 21. XI. Agglutinationsprüfung. Tier Nr. 30 erhielt danach $10 \mathrm{mg}$ Pilocarpin. hydrochlor. Am nächsten Tag beide Tiere je $1 / 3$ Öse Typhusbazillen. Tier Nr. 30 erhielt außerdem $10 \mathrm{mg}$ Pilocarpin. hydrochlor. Am nächsten Tag die gleiche Dosis. Am 25. XI. bei beiden Tieren Agglutinationsprüfung, die am 28. XI. wiederholt wurde (vgl. Tabelle 16). 
Tabelle 16.

\begin{tabular}{|c|c|c|c|c|c|c|c|}
\hline \multirow[b]{2}{*}{ Datum } & \multirow[b]{2}{*}{$\begin{array}{c}\text { Ver- } \\
\text { dünnung }\end{array}$} & \multicolumn{2}{|c|}{ Vor der Injektion } & \multicolumn{4}{|c|}{ Nach der Injektion } \\
\hline & & $\begin{array}{c}\text { Nr. } 29 \\
2,35 \mathrm{~kg} \text { Gewicht } \\
\text { Kontrolle }\end{array}$ & $\begin{array}{c}\text { Nr. } 30 \\
2,80 \mathrm{~kg} \text { Gewicht } \\
\text { Pilokarpin }\end{array}$ & $\begin{array}{r}25 . \\
\text { Nr. } 29\end{array}$ & $\begin{array}{l}\text { IX. } \\
\text { Nr. } 30\end{array}$ & $\begin{array}{r}28 . \\
\text { Nr. } 29\end{array}$ & $\begin{array}{l}\text { IX. } \\
\text { Nr. } 30\end{array}$ \\
\hline 21. bis & $1: 160$ & + & + & + & + & + & + \\
\hline 28. XI. & $1: 320$ & + & + & + & + & + & + \\
\hline & $1: 640$ & + & + & + & + & + & + \\
\hline & $1: 1280$ & + & + & + & + & + & + \\
\hline & $1: 2560$ & + & + & + & 0 & + & + \\
\hline & $1: 3200$ & 0 & 0 & + & 0 & + & 0 \\
\hline & $1: 5120$ & 0 & 0 & 0 & 0 & 0 & 0 \\
\hline
\end{tabular}

Durch diese Modifikation in der Versuchsanordnung, wobei das eine Tier $3 \mathrm{mal}$ Pilokarpin erhielt (vor und nach der Injektion des Antigens), haben wir eine Abnahme, 3 Tage später eine relative $\mathbf{Z u}-$ nahme des Agglatiningehaltes, der aber nicht größer ist als vor der Pilokarpininjektion. Das Kontrolltier zeigt am 25. XI. eine Zunahme des Agglutiningehaltes.

In weiteren Versuchen erhielten die Tiere nach der Antigeninjektion täglich Pilokarpin in verschiedenen Mengen, wobei wir uns von dem Gedanken leiten ließen, ob nicht vielleicht die wiederholten Pilokarpininjektionen zu einer vermehrten Antikörperbildung ftubren.

\section{Versueh 5.}

Vier Kaninchen erhielten am 22. IX. Typhusbażillen wie in Versuch 1. Tier Nr. 9 diente als Kontrolle. Nr. 12 erbielt 1 Woche lang täglich subkutan $5 \mathrm{mg}$ and $\mathrm{Nr} .8$ and 10 intravenös $2 \mathrm{mg}$ Pilocarpin. hydrochlor. Nur Kaninchen Nr. 9 zeigte einen normalen Agglutiningehalt von 1:40. Die Agglutinationsprüfung am 30. IX. ergab folgendes:

Tabelle 17.

\begin{tabular}{c|c|c|c|c|c}
\hline & & \multicolumn{4}{|c|}{ Körpergewicht } \\
Datum & Verdünnung & $\begin{array}{c}\text { Nr. 8 } \\
2,60 \mathrm{~kg} \\
\text { intravenös }\end{array}$ & $\begin{array}{c}\text { Nr. 10 } \\
\mathbf{3 , 0 0} \mathbf{~ k g}\end{array}$ & $\begin{array}{c}\text { Nr. 12 } \\
2,40 \mathrm{~kg} \\
\text { subkutan }\end{array}$ & $\begin{array}{c}\text { Nr. 9 } \\
2,30 \mathrm{~kg} \\
\text { Kontrolle }\end{array}$ \\
\hline \multirow{3}{*}{ 30. IX. } & $1: 40$ & + & + & + & + \\
& $1: 80$ & + & + & + & + \\
& $1: 160$ & + & + & + & + \\
& $1: 320$ & + & + & + & + \\
& $1: 640$ & + & + & + & + \\
& $-1: 1280$ & 0 & 0 & 0 & + \\
& $1: 2560$ & 0 & 0 & 0 & 0 \\
& $1: 5120$ & 0 & 0 & 0 & 0
\end{tabular}


Das Kontrolltier zeigt einen höheren Agglutiningehalt als die drei Pilokarpintiere, wobei allerdings za bertucksichtigen ist, daß dieses Tier schon normal einen geringen Agglutiningehalt zeigte. Am 30. IX. erhielten alle Tiere eine neue Antigeninjektion und danach in der 2. Woche Kaninchen Nr. 12 täglich subkutan $8 \mathrm{mg}$, Kaninchen Nr. 8 und 10 intravenös $4 \mathrm{mg}$ Pilocarpin. hydrochlor. Die 2. Agglutinationsprufung am 7. X. ergab:

Tabelle 18.

\begin{tabular}{l|l|l|l|c|c}
\hline Datum & Verdünnung & \multicolumn{2}{|c|}{$\begin{array}{c}\text { Nr. 8 } \\
\text { intravenös }\end{array}$} & $\begin{array}{c}\text { Nr. 10 } \\
\text { subkutan }\end{array}$ & $\begin{array}{c}\text { Nr. 12 } \\
\text { Kontrolle }\end{array}$ \\
\hline \multirow{2}{*}{. X. } & $1: 80$ & + & + & + & + \\
& $1: 160$ & + & + & + & + \\
& $1: 320$ & + & + & + & + \\
& $1: 640$ & + & + & + & + \\
& $1: 1280$ & + & + & + & + \\
& $1: 2560$ & \pm & 0 & + & + \\
& $1: 5120$ & 0 & 0 & 0 & + \\
& $1: 10240$ & 0 & 0 & 0 & + \\
& $1: 20480$ & 0 & 0 & 0 & 0
\end{tabular}

Am 7. X. erhielten alle Tiere wieder eine Antigeninjektion und in der darauffolgenden Woche Kaninchen Nr. 12 täglich subkutan $10 \mathrm{mg}$, Kaninchen Nr. 8 und 10 intravenös $5 \mathrm{mg}$ Pilocarpin. hydrochlor. Die Agglutinationsprifung am 14. X. ergab:

Tabelle 19.

\begin{tabular}{l|l|c|c|c|c}
\hline Datum & Verdünnung & \multicolumn{2}{|c|}{$\begin{array}{c}\text { Nr. 8 } \\
\text { intravenös }\end{array}$} & $\begin{array}{c}\text { Nr. 10 } \\
\text { subkutan }\end{array}$ & $\begin{array}{c}\text { Nr. 12 } \\
\text { Kontrolle }\end{array}$ \\
\hline \multirow{2}{*}{ 14. X. } & $1: 160$ & + & + & + & + \\
& $1: 320$ & + & + & + & + \\
& $1: 640$ & + & + & + & + \\
& $1: 1280$ & + & + & + & + \\
& $1: 2560$ & + & 0 & + & + \\
& $1: 5120$ & + & 0 & + & + \\
& $1: 10240$ & 0 & 0 & 0 & + \\
& $1: 20480$ & 0 & 0 & 0 & + \\
& $1: 40560$ & 0 & 0 & 0 & 0
\end{tabular}

Auch hier weist das Kontrolltier einen höheren Agglutiningehalt auf als die mit Pilokarpin behandelten. 
Versuch 6.

Sechs Kaninchen erhielten am 20. X. Typhusbakterien, nachdem die normale Agglutination festgestellt wurde (Tabelle 20).

Tabelle 20.

\begin{tabular}{|c|c|c|c|c|c|c|c|}
\hline \multirow{3}{*}{ Datum } & \multirow{3}{*}{$\begin{array}{c}\text { Ver- } \\
\text { dünnung }\end{array}$} & \multicolumn{6}{|c|}{ Körpergewicht } \\
\hline & & $\begin{array}{r}\text { Nr. } 21 \\
2,10 \mathrm{~kg}\end{array}$ & $\begin{array}{c}\mathrm{Nr} .22 \\
2.55 \mathrm{~kg}\end{array}$ & $\begin{array}{c}\mathrm{Nr}: 24 \\
2.90 \mathrm{~kg}\end{array}$ & $\begin{array}{l}\text { Nr. } 23 \\
2.25 \mathrm{~kg}\end{array}$ & $\begin{array}{l}\text { Nr. } 25 \\
2,75 \mathrm{~kg}\end{array}$ & $\begin{array}{c}\mathrm{Nr} .26 \\
2.85 \mathrm{~kg}\end{array}$ \\
\hline & & \multicolumn{3}{|c|}{ Kontrolltiere } & \multicolumn{3}{|c|}{ Versuchstiere } \\
\hline $20 . X$. & $1: 5$ & + & + & + & + & + & 0 \\
\hline & $1: 10$ & + & + & + & + & + & 0 \\
\hline & $1: 20$ & + & + & 0 & 0 & + & 0 \\
\hline & $1: 40$ & + & 0 & 0 & 0 & 0 & 0 \\
\hline
\end{tabular}

In der darauffolgenden Woche erhielten die Tiere Nr. 23-26 intravenös täglich $2 \mathrm{mg}$ Pilocarpin. hydrochlor. Die Agglutinationsprufung am 28. X. ergab folgendes:

Tabelle 21.

\begin{tabular}{l|l|c|c|c|c|c|c}
\hline \multirow{2}{*}{ Datum } & Ver- & \multicolumn{4}{|c|}{ Kontrolltiere } & \multicolumn{3}{c}{ Pilokarpintiere } \\
& dünnung & Nr. 21 & Nr. 22 & Nr. 24 & Nr. 23 & Nr. 25 & Nr. 26. \\
\hline \multirow{2}{*}{ 28. X. } & $1: 80$ & + & + & + & + & + & + \\
& $1: 160$ & + & + & + & + & + & + \\
& $1: 320$ & + & + & + & + & + & + \\
& $1: 640$ & + & + & + & + & + & + \\
& $1: 1280$ & + & + & + & + & + & + \\
& $1: 2560$ & + & + & + & + & + & 0 \\
$1: 5120$ & 0 & + & 0 & 0 & + & 0 \\
& $1: 10240$ & 0 & 0 & 0 & 0 & 0 & 0
\end{tabular}

Zwischen dem Kontrolltier Nr. 22 und dem Pilokarpintier Nr. 25 ist kein Unterschied festzustellen, auch der normale Agglutiningehalt beider. Tiere vor der Antigeninjektion war der gleiche. Die tubrigen Tiere zeigen geringfigige Unterschiede.

28. $\mathrm{X}$. bei allen Tieren eine 2. Antigeninjektion. Tier Nr. 21 und 24 (Kontrolltiere) starben in den nächsten Tagen. Tier Nr. 23, 25 und 26 erhielten in der darauffolgenden Woche täglich $10 \mathrm{mg}$ Pilocarpin. hydrochlor. intravenös. Am 4. XI. ergab die Agglutinationsprïung folgendes: 
Über den Einfluß von Atropin und Pilokarpin usw.

Tabelle 22.

\begin{tabular}{l|l|c|c|c|c}
\hline \multirow{2}{*}{ Datum } & Verdïnnung & $\begin{array}{c}\text { Kontrolltier } \\
\text { Nr. 22 }\end{array}$ & \multicolumn{4}{|c}{ Versuchstiere } \\
& Nr. 23 & Nr. 25 & Nr. 26 \\
\hline \multirow{2}{*}{ 4. XI. } & $1: 160$ & + & + & + & + \\
& $1: 320$ & + & + & + & + \\
& $1: 640$ & + & + & + & + \\
& $1: 1280$ & + & + & + & + \\
& $1: 2560$ & + & + & + & + \\
& $1: 5120$ & + & 0 & + & 0 \\
& $1: 10240$ & 0 & 0 & 0 & 0
\end{tabular}

Auch hier zeigt das Kontrolltier Nr. 22 gegenüber dem Pilokarpintier Nr. 25 keinen. Unterschied des Agglutiningehaltes. Am 4. XI. erhielten die Tiere eine 3. Antigeninjektion und in den nächsten Tagen Tier Nr. 23, 25 und 26 je $5 \mathrm{mg}$ Pilocarpin. hydrochlor. intravenös. Am 8. XI. Tier Nr. 23 und 25 je $10 \mathrm{mg}$ Pilocarpin. hydrochlor. intravenös. Tier Nr. $2615 \mathrm{mg}$. Das Kontrolltier erhielt $1 \mathrm{cem}$ phys. NaCl. Die Agglutinationsprüfung des vor und nach der Pilokarpinzufuhr und 1 Stunde später entnommenen Blutes zeigte folgendes:

Tabelle 23.

\begin{tabular}{|c|c|c|c|c|c|c|c|c|c|}
\hline \multirow{3}{*}{$\begin{array}{l}\text { Datum } \\
\text { 8. XI. }\end{array}$} & \multirow{3}{*}{$\begin{array}{c}\begin{array}{c}\text { Ver- } \\
\text { dünnung }\end{array} \\
1: 160\end{array}$} & \multirow{2}{*}{$\begin{array}{r}\text { Vo } \\
\mathrm{Nr} .22 \\
\text { Kontrolle } \\
\end{array}$} & \multirow{2}{*}{\multicolumn{3}{|c|}{$\begin{array}{l}\text { der Injektion } \\
\mid \begin{array}{c}\mathrm{Nr} .23|\mathrm{Nr} .25| \mathrm{Nr} .26 \\
\text { Versuchstiere }\end{array}\end{array}$}} & \multicolumn{4}{|c|}{ Nach der Injektion } \\
\hline & & & & & & $\begin{array}{c}\text { Nr. } 22 \\
\text { Kontrolle }\end{array}$ & $\begin{array}{r}\text { Nr. } 2 \\
V\end{array}$ & & $\begin{array}{l}\text { Tr. } 26 \\
\mathrm{e}\end{array}$ \\
\hline & & + & + & + & + & + & + & + & + \\
\hline & $1: 320$ & + & + & + & + & + & + & + & + \\
\hline & $1: 640$ & + & + & + & + & + & + & + & + \\
\hline & $1: 1280$ & + & + & + & + & + & + & + & + \\
\hline & $1: 2560$ & + & + & + & + & + & + & + & + \\
\hline & $1: 5120$ & + & + & + & 0 & 0 & + & 0 & 0 \\
\hline & $1: 10240$ & 0 & 0 & 0 & 0 & 0 & 0 & 0 & 0 \\
\hline
\end{tabular}

Wenn wir die Mittelzahlen ans den vorhergehenden Tabellen in ein Koordinatensystem eintragen, wie in Kurve 1 (vgl. Kurve 2), so

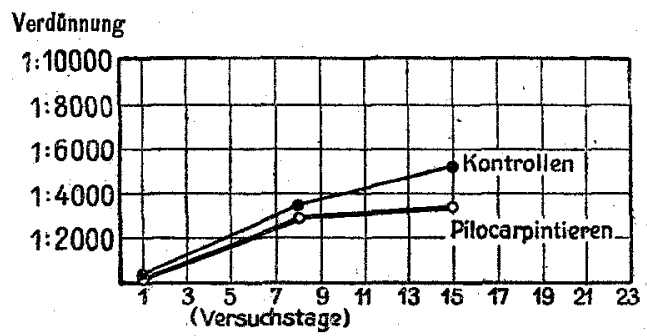

Kurve 2. 
sehen wir, daß ein Einfluß des Pilokarpins auf dẹn Antikörpergehalt des Serums nieht festzustellen ist.

Wir haben im vorstehenden versucht, eine vollkommen objektive Darstellung unserer Versuche zu geben. Wir glauben, daß sie weder für das Atropin noch für das Pilokarpin eine regelmäßige und deutliche Beeinflussung der Agglutininbildung ergeben. Wenn auch damit die Resultate von Salomonsen und Madsen nicht widerlegt sind, weil diese Autoren an einem Pferd experimentiert haben und wir an Kaninchen, so mahnen sie doch zur Vorsicht in der Verallgemeinerung der Ergebnisse dieser Autoren. Insbesondere ist es uns nicht gelungen, durch Pilokarpin eine Steigerung der Agglutininbildung hervorzurufen, während man ans unseren Versuchen vielleicht den Schlaß ziehen könnte, daß das Atropin eine Abnahme der Agglatininbildung hervorruft. Es ist zu beachten, daß das, was wir Agglutinin nennen, vielleicht gar kein Körper ist im Sinne der Chemie, wie die aus den Drisen der Magenschleimhant sezernierte Salzsäure, deren Sekretion wir durch Atropin and Pilokarpin beeinflussen können, sondern eine physikalische Änderung des Serums. 